University of Texas at El Paso

ScholarWorks@UTEP

\title{
8-2015
}

\section{Invariance Explains Multiplicative and Exponential Skedactic Functions}

Vladik Kreinovich

The University of Texas at El Paso, vladik@utep.edu

Olga Kosheleva

The University of Texas at El Paso, olgak@utep.edu

Hung T. Nguyen

New Mexico State University - Main Campus, hunguyen@nmsu.edu

Songsak Sriboonchitta

Chiang Mai University, songsakecon@gmail.com

Follow this and additional works at: https://scholarworks.utep.edu/cs_techrep

Part of the Mathematics Commons

Comments:

Technical Report: UTEP-CS-15-63

Published in: Van Nam Huynh, Vladik Kreinovich, and Songsak Sriboonchitta (eds.), Causal Inference in Econometrics, Springer Verlag, Cham, Switzerland, 2016, pp. 119-131.

\section{Recommended Citation}

Kreinovich, Vladik; Kosheleva, Olga; Nguyen, Hung T.; and Sriboonchitta, Songsak, "Invariance Explains Multiplicative and Exponential Skedactic Functions" (2015). Departmental Technical Reports (CS). 967. https://scholarworks.utep.edu/cs_techrep/967 


\title{
Invariance Explains Multiplicative and Exponential Skedactic Functions
}

Vladik Kreinovich, Olga Kosheleva, Hung T. Nguyen, and Songsak Sriboonchitta

\begin{abstract}
In many situation, we have an (approximately) linear dependence between several quantities: $y \approx c+\sum_{i=1}^{n} a_{i} \cdot x_{i}$. The variance $v=\sigma^{2}$ of the corresponding approximation error $\varepsilon=y-\left(c+\sum_{i=1}^{n} a_{i} \cdot x_{i}\right)$ often depends on the values of the quantities $x_{1}, \ldots, x_{n}: v=v\left(x_{1}, \ldots, x_{n}\right)$; the function describing this dependence is known as the skedactic function. Empirically, two classes of skedactic functions are most successful: multiplicative functions $v=c \cdot \prod_{i=1}^{n}\left|x_{i}\right|^{\gamma_{i}}$ and exponential functions $v=\exp \left(\alpha+\sum_{i=1}^{n} \gamma_{i} \cdot x_{i}\right)$. In this paper, we use natural invariance ideas to provide a possible theoretical explanation for this empirical success; we explain why in some situations multiplicative skedactic functions work better and in some exponential ones. We also come up with a general class of invariant skedactic function that includes both multiplicative and exponential functions as particular cases.
\end{abstract}

Vladik Kreinovich

Department of Computer Science, University of Texas at El Paso,

500 W. University, El Paso, Texas 79968, USA,

e-mail: vladik@utep.edu

Olga Kosheleva

University of Texas at El Paso, 500 W. University, El Paso, Texas 79968, USA,

e-mail: olgak@utep.edu

Hung T. Nguyen

Department of Mathematical Sciences, New Mexico State University,

Las Cruces, New Mexico 88003, USA, and

Faculty of Economics, Chiang Mai University, Chiang Mai, Thailand,

e-mail: hunguyen@nmsu.edu

Songsak Sriboonchitta

Faculty of Economics, Chiang Mai University, Chiang Mai, Thailand,

e-mail: songsak@econ.chiangmai.ac.th 


\section{Why Are Multiplicative and Exponential Skedactic Functions Empirically Successful: Formulation of the Problem}

Linear dependencies are ubiquitous. In many practical situations, a quantity $y$ depends on several other quantities $x_{1}, \ldots, x_{n}: y=f\left(x_{1}, \ldots, x_{n}\right)$. Often, the ranges of $x_{i}$ are narrow: $x_{i} \approx x_{i}^{(0)}$ for some $x_{i}^{(0)}$, so the differences $\Delta x_{i} \stackrel{\text { def }}{=} x_{i}-x_{i}^{(0)}$ are relatively small. In such situations, we can expand the dependence of $y$ on $x_{i}=x_{i}^{(0)}+\Delta x_{i}$ in Taylor series and keep only linear terms in the resulting expansion:

$$
y=f\left(x_{1}, \ldots, x_{n}\right)=f\left(x_{1}^{(0)}+\Delta x_{1}, \ldots, x_{n}^{(0)}+\Delta x_{n}\right) \approx a_{0}+\sum_{i=1}^{n} a_{i} \cdot \Delta x_{i},
$$

where $a_{0} \stackrel{\text { def }}{=} f\left(x_{1}^{(0)}, \ldots, x_{n}^{(0)}\right)$ and $a_{i} \stackrel{\text { def }}{=} \frac{\partial f}{\partial x_{i}}$. Substituting $\Delta x_{i}=x_{i}-x_{i}^{(0)}$ into this formula, we get

$$
y \approx c+\sum_{i=1}^{n} a_{i} \cdot x_{i}
$$

where $c \stackrel{\text { def }}{=} a_{0}-\sum_{i=1}^{n} a_{i} \cdot x_{i}^{(0)}$.

Linear dependencies are approximate. Usually, in addition to the quantities $x_{1}, \ldots, x_{n}$ that provide the most influence on $y$, there are also many other quantities that (slightly) influence $y$, so many that it is not possible to take all of them into account. Since we do not take these auxiliary quantities into account, the above linear dependence is only approximate.

The corresponding approximation errors $\varepsilon \stackrel{\text { def }}{=} y-\left(c+\sum_{i=1}^{n} a_{i} \cdot x_{i}\right)$ depend on unobserved quantities and thus, cannot be predicted based only on the values of the observed quantities $x_{1}, \ldots, x_{n}$. It is therefore reasonable to view these errors as random variables.

Skedactic functions. A natural way to describe a random variable is by its moments, starting with the mean - the first moment - and the variance - which enables us to compute the second moment. If the first moment is not 0 , i.e., if the linear approximation is biased, we can always correct this bias by appropriately updating the constant $c$.

Next, we need to know the second moment which, since the mean is 0 , coincides with the variance $v$. In general, for different values of $x_{i}$, we may have different values of the variance. For example, in econometrics, if we are trying to predict how investment $x_{1}$ in an industry affects its output $y$, clearly larger investments result result not only in larger output, but also in larger output variations.

The function $v\left(x_{1}, \ldots, x_{n}\right)$ that describes how the variance depends on the values of the quantities $x_{1}, \ldots, x_{n}$ is known as the skedactic function.

Which skedactic functions are empirically successful. In econometric applications, two major classes of skedactic functions have been empirically successful: 
multiplicative functions (see, e.g., [2]; [3], Section 9.3; and [4])

$$
v\left(x_{1}, \ldots, x_{n}\right)=c \cdot \prod_{i=1}^{n}\left|x_{i}\right|^{\gamma_{i}}
$$

and exponential functions ([5], Chapter 8)

$$
v\left(x_{1}, \ldots, x_{n}\right)=\exp \left(\alpha+\sum_{i=1}^{n} \gamma_{i} \cdot x_{i}\right) .
$$

According to the latest review [4]:

- neither of this functions has a theoretical justification, and

- in most situations, the multiplication function results in more accurate estimates.

What we do in this paper. In this paper, we use reasonable invariance ideas to provide a possible theoretical explanation for the empirical success of multiplicative and exponential skedactic functions.

We also use invariance to come up with a more general class of skedactic functions to use when neither multiplicative nor exponential functions provide a sufficiently accurate description of the desired dependence.

\section{Natural Invariances}

Scaling. Many economics quantities correspond to prices, wages, etc. and are therefore expressed in terms of money. The numerical value of such a quantity depends on the choice of a monetary unit. For example, when a European country switches to Euro from its original currency, the actual incomes do not change (at least not immediately), but all the prices and wages get multiplied by the corresponding exchange rate $k: x_{i} \rightarrow x_{i}^{\prime}=k \cdot x_{i}$.

Similarly, quantities that describe the goods, such as amount of oil or amount of sugar, also change their numerical values when we use different units: for example, for the oil production, we get different numerical values when we use barrels and when we use metric tons.

When the numerical value of a quantity gets thus re-scaled (multiplied by a constant), the value of its variance gets multiplied by the square of this constant.

Scale-invariance. Since changing the measuring units for measuring $x_{1}, \ldots, x_{n}$ does not change the corresponding economic situations, it makes sense to require that the skedactic function also does not change under such re-scaling: namely, for each combination of re-scalings on inputs, there should be an appropriate re-scaling of the output after which the dependence remains the same.

In precise terms, this means that for every combination of numbers $k_{1}, \ldots, k_{n}$, there should exist a value $k=k\left(k_{1}, \ldots, k_{n}\right)$ with the following property: 
$v=v\left(x_{1}, \ldots, x_{n}\right)$ if and only if $v^{\prime}=v\left(x_{1}^{\prime}, \ldots, x_{n}^{\prime}\right)$, where $v^{\prime}=k \cdot v$ and $x_{i}^{\prime}=k_{i} \cdot x_{i}$.

Shift and shift-invariance. While most economic quantities are scale-invariant, some are not: e.g., the unemployment rate is measured in percents, there is a fixed unit. Many such quantities, however, can have different numerical values depending on how we define a starting point.

For example, we can measure unemployment in absolute units, or we can measure it by considering the difference $x_{i}-k_{i}$ between the actual unemployment and the ideal level $k_{i}>0$ which, in the opinion of the economists, corresponds to full employment.

In general, for such quantities, we have a shift transformation $x_{i} \rightarrow x_{i}^{\prime}=x_{i}+k_{i}$. To consider dependence on such quantities, it is therefore reasonable to consider skedactic functions which are shift-invariant, i.e., for which for every combinations of numbers $\left(k_{1}, \ldots, k_{n}\right)$, there exists a number $k$ for which

$$
v=v\left(x_{1}, \ldots, x_{n}\right) \text { if and only if } v^{\prime}=f\left(x_{1}^{\prime}, \ldots, x_{n}^{\prime}\right) \text {, where } v^{\prime}=k \cdot v \text { and } x_{i}^{\prime}=x_{i}+k_{i} \text {. }
$$

\section{Case of Scale Invariance: Definitions and the Main Result}

Definition 1. We say that a non-negative measurable function $v\left(x_{1}, \ldots, x_{n}\right)$ is scaleinvariant if for every $n$-tuple of real numbers $\left(k_{1}, \ldots, x_{n}\right)$, there exists a real number $k=k\left(k_{1}, \ldots, k_{n}\right)$ for which, for every $x_{1}, \ldots, x_{n}$ and $v$, the following two conditions are equivalent to each other:

- $v=v\left(x_{1}, \ldots, x_{n}\right)$;

- $v^{\prime}=v\left(x_{1}^{\prime}, \ldots, x_{n}^{\prime}\right)$, where $v^{\prime}=k \cdot v$ and $x_{i}^{\prime}=k_{i} \cdot x_{i}$.

Proposition 1. A skedactic function is scale-invariant if and only it has the form $v\left(x_{1}, \ldots, x_{n}\right)=c \cdot \prod_{i=1}^{n}\left|x_{i}\right|^{\gamma_{i}}$ for some values $c$ and $\gamma_{i}$.

Comment. For reader's convenience, all the proofs are placed in the last Proofs section.

Discussion. Thus, scale-invariance explains the use of multiplicative skedactic functions.

\section{Case of Shift-Invariance: Definitions and the Main Result}

Definition 2. We say that a non-zero non-negative measurable function $v\left(x_{1}, \ldots, x_{n}\right)$ is shift-invariant if for every $n$-tuple of real numbers $\left(k_{1}, \ldots, x_{n}\right)$, there exists a real number $k=k\left(k_{1}, \ldots, k_{n}\right)$ for which, for every $x_{1}, \ldots, x_{n}$ and $v$, the following two conditions are equivalent to each other: 
- $v=v\left(x_{1}, \ldots, x_{n}\right)$;

- $v^{\prime}=v\left(x_{1}^{\prime}, \ldots, x_{n}^{\prime}\right)$, where $v^{\prime}=k \cdot v$ and $x_{i}^{\prime}=x_{i}+k_{i}$.

Proposition 2. A skedactic function is scale-invariant if and only it has the form $v\left(x_{1}, \ldots, x_{n}\right)=\exp \left(\alpha+\prod_{i=1}^{n} \gamma_{i} \cdot x_{i}\right)$ for some values $\alpha$ and $\gamma_{i}$.

Discussion. Thus, shift-invariance explains the use of exponential skedactic functions. The fact that most economic quantities are scale-invariant explains why, in general, multiplicative skedactic functions are more empirically successful.

\section{General Case}

General case: discussion. A general case is when some of the inputs are scaleinvariant and some are shift-invariant. Without losing generality, let us assume that the first $m$ variables $x_{1}, \ldots, x_{m}$ are scale-invariant, while the remaining variables $x_{m+1}, \ldots, x_{n}$ are shift-invariant.

Definition 3. Let $m \leq n$ be an integer. We say that a non-zero non-negative measurable function $v\left(x_{1}, \ldots, x_{n}\right)$ is $m$-invariant if for every $n$-tuple of real numbers $\left(k_{1}, \ldots, x_{n}\right)$, there exists a real number $k=k\left(k_{1}, \ldots, k_{n}\right)$ for which, for every $x_{1}, \ldots, x_{n}$ and $v$, the following two conditions are equivalent to each other:

- $v=v\left(x_{1}, \ldots, x_{n}\right)$;

- $v^{\prime}=v\left(x_{1}^{\prime}, \ldots, x_{n}^{\prime}\right)$, where $v^{\prime}=k \cdot v, x_{i}^{\prime}=k_{i} \cdot x_{i}$ for $i \leq m$, and $x_{i}^{\prime}=x_{i}+k_{i}$ for $i>m$.

Proposition 3. A skedactic function is m-invariant if and only it has the form

$$
v\left(x_{1}, \ldots, x_{n}\right)=\exp \left(\alpha+\sum_{i=1}^{m} \gamma_{i} \cdot \ln \left(\left|x_{i}\right|\right)+\sum_{i=m+1}^{n} \gamma_{i} \cdot x_{i}\right)
$$

for some values $\mu$ and $\gamma_{i}$.

Discussion. For $m=n$, this formula leads to a multiplicative skedactic function, with $c=\exp (\alpha)$. For $m=0$, this formula leads to the exponential skedactie function. For intermediate values $m=1,2, \ldots, n-1$, we get new expressions that may be useful when neither multiplicative not exponential skedactic functions work well.

\section{Proofs}

Proof of Proposition 1. It is easy to check that the multiplicative skedactic function is indeed scale-invariant: we can take $k=\prod_{i=1}^{n}\left|k_{i}\right|^{\gamma_{i}}$. 
Let us prove that, vice versa, if a skedactic function is scale-invariant, then it is multiplicative. Indeed, the above equivalence condition means that for every $k_{1}, \ldots, k_{n}, v=v\left(x_{1}, \ldots, x_{n}\right)$ implies that $v^{\prime}=v\left(x_{1}^{\prime}, \ldots, x_{n}^{\prime}\right)$, where $v^{\prime}=k \cdot v$ and $x_{i}^{\prime}=$ $k_{i} \cdot x_{i}$. Substituting the expressions for $v^{\prime}$ and $k_{i}^{\prime}$ into the equality $v^{\prime}=v\left(x_{1}^{\prime}, \ldots, x_{n}^{\prime}\right)$, we conclude that $k \cdot v=v\left(k_{1} \cdot x_{1}, \ldots, k_{n} \cdot x_{n}\right)$.

We know that $k=k\left(k_{1}, \ldots, k_{n}\right)$ and $v=v\left(x_{1}, \ldots, x_{n}\right)$. Thus, we conclude that

$$
k\left(k_{1}, \ldots, k_{n}\right) \cdot v\left(x_{1}, \ldots, x_{n}\right)=v\left(k_{1} \cdot x_{1}, \ldots, k_{n} \cdot x_{n}\right) .
$$

From this equation, we infer that

$$
k\left(k_{1}, \ldots, k_{n}\right)=\frac{v\left(k_{1} \cdot x_{1}, \ldots, k_{n} \cdot x_{n}\right)}{v\left(x_{1}, \ldots, x_{n}\right)} .
$$

The right-hand side of this formula is a non-negative measurable function, so we can conclude that the ratio $k\left(k_{1}, \ldots, k_{n}\right)$ is also non-negative and measurable.

Let us now consider two different tuples $\left(k_{1}, \ldots, k_{n}\right)$ and $\left(k_{1}^{\prime}, \ldots, k_{n}^{\prime}\right)$. If we first use the first re-scaling, i.e., go from $x_{i}$ to $x_{i}^{\prime}=k_{i} \cdot x_{i}$, we get

$$
v\left(x_{1}^{\prime}, \ldots, x_{n}^{\prime}\right)=v\left(k_{1} \cdot x_{1}, \ldots, k_{n} \cdot x_{n}\right)=k\left(k_{1}, \ldots, k_{n}\right) \cdot v\left(x_{1}, \ldots, x_{n}\right) .
$$

If we then apply, to the new values $x_{i}^{\prime}$, an additional re-scaling $x_{i}^{\prime} \rightarrow x_{i}^{\prime \prime}=k_{i}^{\prime} \cdot x_{i}^{\prime}$, we similarly conclude that

$$
v\left(x_{1}^{\prime \prime}, \ldots, x_{n}^{\prime \prime}\right)=v\left(k_{1}^{\prime} \cdot x_{1}^{\prime}, \ldots, k_{n}^{\prime} \cdot x_{n}^{\prime}\right)=k\left(k_{1}^{\prime}, \ldots, k_{n}^{\prime}\right) \cdot v\left(x_{1}^{\prime}, \ldots, x_{n}^{\prime}\right) .
$$

Substituting the expression (6) for $v\left(x_{1}^{\prime}, \ldots, x_{n}^{\prime}\right)$ into this formula, we conclude that

$$
v\left(x_{1}^{\prime \prime}, \ldots, x_{n}^{\prime \prime}\right)=k\left(k_{1}^{\prime}, \ldots, k_{n}^{\prime}\right) \cdot k\left(k_{1}, \ldots, k_{n}\right) \cdot v\left(x_{1}, \ldots, x_{n}\right) .
$$

On the other hand, we could get the values $x_{i}^{\prime \prime}$ if we directly multiply each value $x_{i}$ by the product $k_{i}^{\prime \prime} \stackrel{\text { def }}{=} k_{i}^{\prime} \cdot k_{i}$ :

$$
x_{i}^{\prime \prime}=k_{i}^{\prime} \cdot x_{i}^{\prime}=k_{i}^{\prime} \cdot\left(k_{i} \cdot x_{i}\right)=\left(k_{i}^{\prime} \cdot k_{i}\right) \cdot x_{i}=k_{i}^{\prime \prime} \cdot x_{i} .
$$

For the new values $k_{i}^{\prime \prime}$, the formula (6) takes the form

$$
v\left(x_{1}^{\prime \prime}, \ldots, x_{n}^{\prime \prime}\right)=k\left(k_{1}^{\prime \prime}, \ldots, k_{n}^{\prime \prime}\right) \cdot v\left(x_{1}, \ldots, x_{n}\right)=k\left(k_{1}^{\prime} \cdot k_{1}, \ldots, k_{n}^{\prime} \cdot k_{n}\right) \cdot v\left(x_{1}, \ldots, x_{n}\right) .
$$

The left-hand sides of the formulas (8) and (9) and the same, hence the right-hand sides are also equal, i.e.,

$$
k\left(k_{1}^{\prime} \cdot k_{1}, \ldots, k_{n}^{\prime} \cdot k_{n}\right) \cdot v\left(x_{1}, \ldots, x_{n}\right)=k\left(k_{1}^{\prime}, \ldots, k_{n}^{\prime}\right) \cdot k\left(k_{1}, \ldots, k_{n}\right) \cdot v\left(x_{1}, \ldots, x_{n}\right) .
$$

If the skedactic function is always equal to 0 , then it is multiplicative, with $c=0$. If it is not everywhere 0 , this means that its value is different from 0 for some combination of values $x_{1}, \ldots, x_{n}$. Substituting these values into the formula (10) and 
dividing both sides by $v\left(x_{1}, \ldots, x_{n}\right) \neq 0$, we conclude that

$$
k\left(k_{1}^{\prime} \cdot k_{1}, \ldots, k_{n}^{\prime} \cdot k_{n}\right)=k\left(k_{1}^{\prime}, \ldots, k_{n}^{\prime}\right) \cdot k\left(k_{1}, \ldots, k_{n}\right) .
$$

When $k_{i}=k_{i}^{\prime}=-1$ for some $i$ and $k_{i}^{\prime}=k_{i}=1$ for all other $i$, we get

$$
1=k(1, \ldots, 1)=k\left(k_{1}, \ldots, k_{n}\right) \cdot k\left(k_{1}, \ldots, k_{n}\right)=k^{2}\left(k_{1}, \ldots, k_{n}\right) .
$$

Since the function $k_{i}$ is non-negative, this means that $k\left(k_{1}, \ldots, k_{n}\right)=1$. Thus, from the formula (11), we can conclude that the value $k\left(k_{1}, \ldots, k_{n}\right)$ does not change if we change the signs of $k_{i}$, i.e., that

$$
k\left(k_{1}, \ldots, k_{n}\right)=k\left(\left|k_{1}\right|, \ldots,\left|k_{n}\right|\right) .
$$

Taking logarithms of both sides of the formula (11), and taking into account that $\ln \left(a \cdot a^{\prime}\right)=\ln (a)+\ln \left(a^{\prime}\right)$, we conclude that

$$
\ln \left(k\left(k_{1}^{\prime} \cdot k_{1}, \ldots, k_{n}^{\prime} \cdot k_{n}\right)\right)=\ln \left(k\left(k_{1}^{\prime}, \ldots, k_{n}^{\prime}\right)\right)+\ln \left(k\left(k_{1}, \ldots, k_{n}\right)\right) .
$$

Let us now define an auxiliary function

$$
K\left(K_{1}, \ldots, K_{n}\right) \stackrel{\text { def }}{=} \ln \left(k\left(\exp \left(K_{1}\right), \ldots, \exp \left(K_{n}\right)\right) .\right.
$$

Since the function $k\left(k_{1}, \ldots, k_{n}\right)$ is measurable, the function $K\left(K_{1}, \ldots, K_{n}\right)$ is also measurable.

Since $\exp \left(a+a^{\prime}\right)=\exp (a) \cdot \exp \left(a^{\prime}\right)$, we conclude that when $k_{i}=\exp \left(K_{i}\right)$ and $k_{i}^{\prime}=\exp \left(K_{i}^{\prime}\right)$, then $k_{i} \cdot k_{i}^{\prime}=\exp \left(K_{i}\right) \cdot \exp \left(K_{i}^{\prime}\right)=\exp \left(K_{i}+K_{i}^{\prime}\right)$. Thus, from (13), we conclude that for the new function $K\left(K_{1}, \ldots, K_{n}\right)$, we get

$$
K\left(K_{1}^{\prime}+K_{1}, \ldots, K_{n}^{\prime}+K_{n}\right)=K\left(K_{1}^{\prime}, \ldots, K_{n}^{\prime}\right)+K\left(K_{1}, \ldots, K_{n}\right) .
$$

Functions that satisfy the property (14) are known as additive. It is known (see, e.g., [1]) that every measurable additive function is linear, i.e., has the form

$$
K\left(K_{1}, \ldots, K_{n}\right)=\sum_{i=1} \gamma_{i} \cdot X_{i}
$$

for some values $\gamma_{i}$.

From $K\left(K_{1}, \ldots, K_{n}\right)=\ln \left(k\left(\exp \left(K_{1}\right), \ldots, \exp \left(K_{n}\right)\right)\right)$, it follows that

$$
k\left(\exp \left(K_{1}\right), \ldots, \exp \left(K_{n}\right)\right)=\exp \left(K\left(K_{1}, \ldots, K_{n}\right)\right)=\exp \left(\sum_{i=1}^{n} \gamma_{i} \cdot K_{i}\right)
$$

For each $k_{1}, \ldots, k_{n}$, we have

$$
k\left(k_{1}, \ldots, k_{n}\right)=k\left(\left|k_{1}\right|, \ldots,\left|k_{n}\right|\right) .
$$

For $K_{i}=\ln \left(\left|k_{i}\right|\right)$, we have $\exp \left(K_{i}\right)=\left|k_{i}\right|$, hence 


$$
k\left(k_{1}, \ldots, k_{n}\right)=\exp \left(\sum_{i=1}^{n} \gamma_{i} \cdot \ln \left(\left|k_{i}\right|\right)\right)=\prod_{i=1}^{n}\left|k_{i}\right|^{\gamma_{i}} \text {. }
$$

From (4), we can now conclude that

$$
v\left(x_{1}, \ldots, x_{n}\right)=k\left(x_{1}, \ldots, x_{n}\right) \cdot v(1, \ldots, 1) .
$$

Substituting expression (16) for $k\left(x_{1}, \ldots, x_{n}\right)$ into this formula and denoting $c \stackrel{\text { def }}{=}$ $v(1, \ldots, 1)$, we get the desired formula for the multiplicative skedastic function $v\left(x_{1}, \ldots, x_{n}\right)=\left.c \cdot \prod_{i=1}^{n}\left|x_{i}\right|\right|_{i}$. The proposition is proven.

Proof of Proposition 2. It is easy to check that the exponential skedactic function is indeed shift-invariant: we can take $k=\exp \left(\sum_{i=1}^{n} \gamma_{i} \cdot k_{i}\right)$.

Let us prove that, vice versa, if a skedactic function is shift-invariant, then it is exponential. Indeed, the above equivalence condition means that for every $k_{1}, \ldots, k_{n}$, $v=v\left(x_{1}, \ldots, x_{n}\right)$ implies that $v^{\prime}=v\left(x_{1}^{\prime}, \ldots, x_{n}^{\prime}\right)$, where $v^{\prime}=k \cdot v$ and $x_{i}^{\prime}=x_{i}+k_{i}$. Substituting the expressions for $v^{\prime}$ and $k_{i}^{\prime}$ into the equality $v^{\prime}=v\left(x_{1}^{\prime}, \ldots, x_{n}^{\prime}\right)$, we conclude that $k \cdot v=v\left(x_{1}+k_{1}, \ldots, x_{n}+k_{n}\right)$.

We know that $k=k\left(k_{1}, \ldots, k_{n}\right)$ and $v=v\left(x_{1}, \ldots, x_{n}\right)$. Thus, we conclude that

$$
k\left(k_{1}, \ldots, k_{n}\right) \cdot v\left(x_{1}, \ldots, x_{n}\right)=v\left(x_{1}+k_{1}, \ldots, x_{n}+k_{n}\right) .
$$

From this equation, we infer that

$$
k\left(k_{1}, \ldots, k_{n}\right)=\frac{v\left(x_{1}+k_{1}, \ldots, x_{n}+k_{n}\right)}{v\left(x_{1}, \ldots, x_{n}\right)} .
$$

The right-hand side of this formula is a non-negative measurable function, so we can conclude that the ratio $k\left(k_{1}, \ldots, k_{n}\right)$ is also non-negative and measurable.

Let us now consider two different tuples $\left(k_{1}, \ldots, k_{n}\right)$ and $\left(k_{1}^{\prime}, \ldots, k_{n}^{\prime}\right)$. If we first use the first shift, i.e., go from $x_{i}$ to $x_{i}^{\prime}=x_{i}+k_{i}$, we get

$$
v\left(x_{1}^{\prime}, \ldots, x_{n}^{\prime}\right)=v\left(x_{1}+k_{1}, \ldots, x_{n}+k_{n}\right)=k\left(k_{1}, \ldots, k_{n}\right) \cdot v\left(x_{1}, \ldots, x_{n}\right) .
$$

If we then apply, to the new values $x_{i}^{\prime}$, an additional shift $x_{i}^{\prime} \rightarrow x_{i}^{\prime \prime}=x_{i}^{\prime}+k_{i}^{\prime}$, we similarly conclude that

$$
v\left(x_{1}^{\prime \prime}, \ldots, x_{n}^{\prime \prime}\right)=v\left(x_{1}^{\prime}+k_{1}^{\prime}, \ldots, x_{n}^{\prime}+k_{n}^{\prime}\right)=k\left(k_{1}^{\prime}, \ldots, k_{n}^{\prime}\right) \cdot v\left(x_{1}^{\prime}, \ldots, x_{n}^{\prime}\right) .
$$

Substituting the expression (19) for $v\left(x_{1}^{\prime}, \ldots, x_{n}^{\prime}\right)$ into this formula, we conclude that

$$
v\left(x_{1}^{\prime \prime}, \ldots, x_{n}^{\prime \prime}\right)=k\left(k_{1}^{\prime}, \ldots, k_{n}^{\prime}\right) \cdot k\left(k_{1}, \ldots, k_{n}\right) \cdot v\left(x_{1}, \ldots, x_{n}\right) .
$$

On the other hand, we could get the values $x_{i}^{\prime \prime}$ if we directly shift each value $x_{i}$ by the sum $k_{i}^{\prime \prime} \stackrel{\text { def }}{=} k_{i}^{\prime}+k_{i}$ : 


$$
x_{i}^{\prime \prime}=x_{i}^{\prime}+k_{i}^{\prime}=\left(x_{i}+k_{i}\right)+k_{i}^{\prime}=x_{i}+\left(k_{i}+k_{i}^{\prime}\right)=x_{i}+k_{i}^{\prime \prime}
$$

For the new values $k_{i}^{\prime \prime}$, the formula (19) takes the form

$$
\begin{gathered}
v\left(x_{1}^{\prime \prime}, \ldots, x_{n}^{\prime \prime}\right)=k\left(k_{1}^{\prime \prime}, \ldots, k_{n}^{\prime \prime}\right) \cdot v\left(x_{1}, \ldots, x_{n}\right)= \\
k\left(k_{1}+k_{1}^{\prime}, \ldots, k_{n}+k_{n}^{\prime}\right) \cdot v\left(x_{1}, \ldots, x_{n}\right) .
\end{gathered}
$$

The left-hand sides of the formulas (21) and (22) and the same, hence the right-hand sides are also equal, i.e.,

$$
\begin{gathered}
k\left(k_{1}+k_{1}^{\prime}, \ldots, k_{n}+k_{n}^{\prime}\right) \cdot v\left(x_{1}, \ldots, x_{n}\right)= \\
k\left(k_{1}^{\prime}, \ldots, k_{n}^{\prime}\right) \cdot k\left(k_{1}, \ldots, k_{n}\right) \cdot v\left(x_{1}, \ldots, x_{n}\right) .
\end{gathered}
$$

Since the skedactic function is assumed to be non-zero, its value is different from 0 for some combination of values $x_{1}, \ldots, x_{n}$. Substituting these values into the formula (23) and dividing both sides by $v\left(x_{1}, \ldots, x_{n}\right) \neq 0$, we conclude that

$$
k\left(k_{1}+k_{1}^{\prime}, \ldots, k_{n}+k_{n}^{\prime}\right)=k\left(k_{1}^{\prime}, \ldots, k_{n}^{\prime}\right) \cdot k\left(k_{1}, \ldots, k_{n}\right) .
$$

Taking logarithms of both sides of the formula (11), and taking into account that $\ln \left(a \cdot a^{\prime}\right)=\ln (a)+\ln \left(a^{\prime}\right)$, we conclude that

$$
\ln \left(k\left(k_{1}+k_{1}^{\prime}, \ldots, k_{n}+k_{n}^{\prime}\right)\right)=\ln \left(k\left(k_{1}^{\prime}, \ldots, k_{n}^{\prime}\right)\right)+\ln \left(k\left(k_{1}, \ldots, k_{n}\right)\right) .
$$

Thus, the function $\ln \left(k\left(k_{1}, \ldots, k_{n}\right)\right)$ is measurable and additive, and hence ([1]) has the form

$$
\ln \left(k\left(k_{1}, \ldots, k_{n}\right)\right)=\sum_{i=1}^{n} \gamma_{i} \cdot k_{i} .
$$

Hence, by taking exp of both sides, we conclude that

$$
k\left(k_{1}, \ldots, k_{n}\right)=\exp \left(\sum_{i=1}^{n} \gamma_{i} \cdot k_{i}\right) .
$$

From (17), we can now conclude that

$$
v\left(x_{1}, \ldots, x_{n}\right)=k\left(x_{1}, \ldots, x_{n}\right) \cdot v(0, \ldots, 0) .
$$

Substituting expression (26) for $k\left(x_{1}, \ldots, x_{n}\right)$ into this formula and denoting $\alpha \stackrel{\text { def }}{=}$ $\ln (v(0, \ldots, 0))$, so that $v(0, \ldots, 0)=\exp (\alpha)$, we get the desired formula for the exponential skedastic function $v\left(x_{1}, \ldots, x_{n}\right)=\exp \left(\alpha+\sum_{i=1}^{n} \gamma_{i} \cdot x_{i}\right)$. The proposition is proven.

Proof of Proposition 3. It is easy to check that the skedactic function described in the formulation of Proposition 3 is indeed $m$-invariant: we can take 


$$
k=\prod_{i=1}^{m}\left|k_{i}\right|^{\gamma_{i}} \cdot \exp \left(\sum_{i=m+1}^{n} \gamma_{i} \cdot k_{i}\right) \text {. }
$$

Let us prove that, vice versa, if a skedactic function is $m$-invariant, then it has the desired form. Indeed, the above equivalence condition means that for every $k_{1}, \ldots, k_{n}, v=v\left(x_{1}, \ldots, x_{n}\right)$ implies that $v^{\prime}=v\left(x_{1}^{\prime}, \ldots, x_{n}^{\prime}\right)$, where $v^{\prime}=k \cdot v, x_{i}^{\prime}=k_{i} \cdot x_{i}$ for $i \leq m$, and $x_{i}^{\prime}=x_{i}+k_{i}$ for $i>m$. Substituting the expressions for $v^{\prime}$ and $k_{i}^{\prime}$ into the equality $v^{\prime}=v\left(x_{1}^{\prime}, \ldots, x_{n}^{\prime}\right)$, we conclude that

$$
k \cdot v=v\left(k_{1} \cdot x_{1}, \ldots, k_{m} \cdot x_{m}, x_{m+1}+k_{m+1}, \ldots, x_{n}+k_{n}\right) .
$$

We know that $k=k\left(k_{1}, \ldots, k_{n}\right)$ and $v=v\left(x_{1}, \ldots, x_{n}\right)$. Thus, we conclude that

$$
k\left(k_{1}, \ldots, k_{n}\right) \cdot v\left(x_{1}, \ldots, x_{n}\right)=v\left(k_{1} \cdot x_{1}, \ldots, k_{m} \cdot x_{m}, x_{m+1}+k_{m+1}, \ldots, x_{n}+k_{n}\right) .
$$

From this equation, we infer that

$$
k\left(k_{1}, \ldots, k_{n}\right)=\frac{v\left(k_{1} \cdot x_{1}, \ldots, k_{m} \cdot x_{m}, x_{m+1}+k_{m+1}, \ldots, x_{n}+k_{n}\right)}{v\left(x_{1}, \ldots, x_{n}\right)} .
$$

The right-hand side of this formula is a non-negative measurable function, so we can conclude that the ratio $k\left(k_{1}, \ldots, k_{n}\right)$ is also non-negative and measurable.

Let us now consider two different tuples $\left(k_{1}, \ldots, k_{n}\right)$ and $\left(k_{1}^{\prime}, \ldots, k_{n}^{\prime}\right)$. If we first use the transformation corresponding to the first tuple, i.e., go from $x_{i}$ to $x_{i}^{\prime}=k_{i} \cdot x_{i}$ for $i \leq m$ and to $x_{i}^{\prime}=x_{i}+k_{i}$ for $i>m$, we get

$$
\begin{gathered}
v\left(x_{1}^{\prime}, \ldots, x_{n}^{\prime}\right)=v\left(k_{1} \cdot x_{1}, \ldots, k_{m} \cdot x_{m}, x_{m+1}+k_{m+1}, \ldots, x_{n}+k_{n}\right)= \\
k\left(k_{1}, \ldots, k_{n}\right) \cdot v\left(x_{1}, \ldots, x_{n}\right) .
\end{gathered}
$$

If we then apply, to the new values $x_{i}^{\prime}$, an additional transformation $x_{i}^{\prime} \rightarrow x_{i}^{\prime \prime}=k_{i}^{\prime} \cdot x_{i}^{\prime}$ for $i \leq m$ and $x_{i}^{\prime} \rightarrow x_{i}^{\prime \prime}=x_{i}^{\prime}+k_{i}^{\prime}$ for $i>m$, we similarly conclude that

$$
\begin{gathered}
v\left(x_{1}^{\prime \prime}, \ldots, x_{n}^{\prime \prime}\right)=v\left(k_{1}^{\prime} \cdot x_{1}^{\prime}, \ldots, k_{m}^{\prime} \cdot x_{m}^{\prime}, x_{m+1}^{\prime}+k_{m+1}^{\prime}, \ldots, x_{n}^{\prime}+k_{n}^{\prime}\right)= \\
k\left(k_{1}^{\prime}, \ldots, k_{n}^{\prime}\right) \cdot v\left(x_{1}^{\prime}, \ldots, x_{n}^{\prime}\right) .
\end{gathered}
$$

Substituting the expression (29) for $v\left(x_{1}^{\prime}, \ldots, x_{n}^{\prime}\right)$ into this formula, we conclude that

$$
v\left(x_{1}^{\prime \prime}, \ldots, x_{n}^{\prime \prime}\right)=k\left(k_{1}^{\prime}, \ldots, k_{n}^{\prime}\right) \cdot k\left(k_{1}, \ldots, k_{n}\right) \cdot v\left(x_{1}, \ldots, x_{n}\right) .
$$

On the other hand, we could get the values $x_{i}^{\prime \prime}$ if we directly apply to the tuple $x_{i}$ the transformation corresponding to the product $k_{i}^{\prime \prime}=k_{i}^{\prime} \cdot k_{i}$ for $i \leq m$ and to the sum $k_{i}^{\prime \prime}=k_{i}+k_{i}^{\prime}$ for $i>m: \mathrm{b}$

$$
x_{i}^{\prime \prime}=k_{i}^{\prime} \cdot x_{i}^{\prime}=k_{i}^{\prime} \cdot\left(k_{i} \cdot x_{i}\right)=\left(k_{i}^{\prime} \cdot k_{i}\right) \cdot x_{i}=k_{i}^{\prime \prime} \cdot x_{i}
$$

for $i \leq m$ and 


$$
x_{i}^{\prime \prime}=x_{i}^{\prime}+k_{i}^{\prime}=\left(x_{i}+k_{i}\right)+k_{i}^{\prime}=x_{i}+\left(k_{i}+k_{i}^{\prime}\right)=x_{i}+k_{i}^{\prime \prime}
$$

for $i>m$.

For the new values $k_{i}^{\prime \prime}$, the formula (29) takes the form

$$
\begin{gathered}
v\left(x_{1}^{\prime \prime}, \ldots, x_{n}^{\prime \prime}\right)=k\left(k_{1}^{\prime \prime}, \ldots, k_{n}^{\prime \prime}\right) \cdot v\left(x_{1}, \ldots, x_{n}\right)= \\
k\left(k_{1}^{\prime} \cdot k_{1}, \ldots, k_{m}^{\prime} \cdot k_{m}, k_{m+1}+k_{m+1}^{\prime}, \ldots, k_{n}+k_{n}^{\prime}\right) \cdot v\left(x_{1}, \ldots, x_{n}\right) .
\end{gathered}
$$

The left-hand sides of the formulas (31) and (32) and the same, hence the right-hand sides are also equal, i.e.,

$$
\begin{gathered}
k\left(k_{1}^{\prime} \cdot k_{1}, \ldots, k_{m}^{\prime} \cdot k_{m}, k_{m+1}+k_{m+1}^{\prime}, \ldots, k_{n}+k_{n}^{\prime}\right) \cdot v\left(x_{1}, \ldots, x_{n}\right)= \\
k\left(k_{1}^{\prime}, \ldots, k_{n}^{\prime}\right) \cdot k\left(k_{1}, \ldots, k_{n}\right) \cdot v\left(x_{1}, \ldots, x_{n}\right) .
\end{gathered}
$$

Since we assume that the skedactic function is non-zero, its value is different from 0 for some combination of values $x_{1}, \ldots, x_{n}$. Substituting these values into the formula (33) and dividing both sides by $v\left(x_{1}, \ldots, x_{n}\right) \neq 0$, we conclude that

$$
k\left(k_{1}^{\prime} \cdot k_{1}, \ldots, k_{m}^{\prime} \cdot k_{m}, k_{m+1}+k_{m+1}^{\prime}, \ldots, k_{n}+k_{n}^{\prime}\right)=k\left(k_{1}^{\prime}, \ldots, k_{n}^{\prime}\right) \cdot k\left(k_{1}, \ldots, k_{n}\right) .
$$

When $k_{i}=k_{i}^{\prime}=-1$ for some $i \leq m$ and $k_{i}^{\prime}=k_{i}=1$ for all other $i$, we get

$$
1=k(1, \ldots, 1)=k\left(k_{1}, \ldots, k_{n}\right) \cdot k\left(k_{1}, \ldots, k_{n}\right)=k^{2}\left(k_{1}, \ldots, k_{n}\right) .
$$

Since the function $k_{i}$ is non-negative, this means that $k\left(k_{1}, \ldots, k_{n}\right)=1$. Thus, from the formula (34), we can conclude that the value $k\left(k_{1}, \ldots, k_{n}\right)$ does not change if we change the signs of $k_{i}$ for $i \leq m$, i.e., that

$$
k\left(k_{1}, \ldots, k_{m}, k_{m+1}, \ldots, k_{n}\right)=k\left(\left|k_{1}\right|, \ldots,\left|k_{m}\right|, k_{m+1}, \ldots, k_{n}\right) .
$$

Taking logarithms of both sides of the formula (35), and taking into account that $\ln \left(a \cdot a^{\prime}\right)=\ln (a)+\ln \left(a^{\prime}\right)$, we conclude that

$$
\begin{gathered}
\ln \left(k\left(k_{1}^{\prime} \cdot k_{1}, \ldots, k_{m}^{\prime} \cdot k_{m}, k_{m+1}+k_{m+1}^{\prime}, \ldots, k_{n}+k_{n}^{\prime}\right)\right)= \\
\ln \left(k\left(k_{1}^{\prime}, \ldots, k_{n}^{\prime}\right)\right)+\ln \left(k\left(k_{1}, \ldots, k_{n}\right)\right) .
\end{gathered}
$$

Let us now define an auxiliary function

$$
K\left(K_{1}, \ldots, K_{n}\right) \stackrel{\text { def }}{=} \ln \left(k\left(\exp \left(K_{1}\right), \ldots, \exp \left(K_{m}\right), K_{m+1}, \ldots, K_{n}\right)\right) .
$$

Since the function $k\left(k_{1}, \ldots, k_{n}\right)$ is measurable, the function $K\left(K_{1}, \ldots, K_{n}\right)$ is also measurable.

Since $\exp \left(a+a^{\prime}\right)=\exp (a) \cdot \exp \left(a^{\prime}\right)$, for $i \leq m$, we conclude that when $k_{i}=$ $\exp \left(K_{i}\right)$ and $k_{i}^{\prime}=\exp \left(K_{i}^{\prime}\right)$, then $k_{i} \cdot k_{i}^{\prime}=\exp \left(K_{i}\right) \cdot \exp \left(K_{i}^{\prime}\right)=\exp \left(K_{i}+K_{i}^{\prime}\right)$. Thus, from (36), we conclude that for the new function $K\left(K_{1}, \ldots, K_{n}\right)$, we get 


$$
K\left(K_{1}^{\prime}+K_{1}, \ldots, K_{n}^{\prime}+K_{n}\right)=K\left(K_{1}^{\prime}, \ldots, K_{n}^{\prime}\right)+K\left(K_{1}, \ldots, K_{n}\right) .
$$

The function $K\left(K_{1}, \ldots, K_{n}\right)$ is measurable and additive and hence [1] has the form

$$
K\left(K_{1}, \ldots, K_{n}\right)=\sum_{i=1}^{n} \gamma_{i} \cdot X_{i}
$$

for some values $\gamma_{i}$.

From

$$
K\left(K_{1}, \ldots, K_{n}\right)=\ln \left(k\left(\exp \left(K_{1}\right), \ldots, \exp \left(K_{m}\right), K_{m+1}, \ldots, K_{n}\right)\right)
$$

it follows that

$$
k\left(\exp \left(K_{1}\right), \ldots, \exp \left(K_{m}\right), K_{m+1}, \ldots, K_{n}\right)=\exp \left(K\left(K_{1}, \ldots, K_{n}\right)\right)=\exp \left(\sum_{i=1}^{n} \gamma_{i} \cdot K_{i}\right) .
$$

For each $k_{1}, \ldots, k_{n}$, we have

$$
k\left(k_{1}, \ldots, k_{m}, k_{m+1}, \ldots, k_{n}\right)=k\left(\left|k_{1}\right|, \ldots,\left|k_{m}\right|, k_{m+1}, \ldots, k_{n}\right) .
$$

Let us take $K_{i}=\ln \left(\left|k_{i}\right|\right)$ for $i \leq m$ and $K_{i}=k_{i}$ for $i>m$, then we have $\exp \left(K_{i}\right)=\left|k_{i}\right|$ for $i \leq m$ and $K_{i}=k_{i}$ for $i>m$. Hence,

$$
k\left(k_{1}, \ldots, k_{n}\right)=\exp \left(\sum_{i=1}^{m} \gamma_{i} \cdot \ln \left(\left|k_{i}\right|\right)+\sum_{i=m+1}^{n} \gamma_{i} \cdot k_{i}\right) .
$$

From (27), we can now conclude that

$$
v\left(x_{1}, \ldots, x_{n}\right)=k\left(x_{1}, \ldots, x_{n}\right) \cdot v(1, \ldots, 1,0, \ldots, 0) .
$$

Substituting expression (39) for $k\left(x_{1}, \ldots, x_{n}\right)$ into this formula, we get the desired formula for the skedastic function

$$
v\left(x_{1}, \ldots, x_{n}\right)=\exp \left(\alpha+\sum_{i=1}^{m} \gamma_{i} \cdot \ln \left(\left|x_{i}\right|\right)+\sum_{i=m+1}^{n} \gamma \cdot x_{i}\right),
$$

with $\alpha=\ln (v(1, \ldots, 1,0, \ldots, 0))$. The proposition is proven.

\section{Acknowledgments}

We acknowledge the partial support of the Center of Excellence in Econometrics, Faculty of Economics, Chiang Mai University, Thailand. 
This work was also supported in part by the National Science Foundation grants HRD-0734825 and HRD-1242122 (Cyber-ShARE Center of Excellence) and DUE0926721.

\section{References}

1. J. Aczél and J. Dhombres, Functional Equations in Several Variables, Cambridge University Press, 2008.

2. A. C. Harvey, "Estimating regression models with multiplicative heteroscedasticity", Econometrica, 1976, Vol. 44, pp. 461-465.

3. G. G. Judge, R. C. Hill, W. E. Griffiths, H. Lütkepohl, and T.-C.Lee, Introduction to the Theory and Practice of Econometrics, John Wiley \& Sons, New York, 1988.

4. J. P. Romano and M. Wolf, Resurrecting Weighted Least Squares, University of Zurich, Department of Economics, Working Paper No. 172, August 2015, available at SSRN http://wwrn.com/abstract=2491081.

5. J. M. Wooldridge, Introductory Econometrics, South-Western, Mason, Ohio, 2012. 